\title{
A comparison of trunk and lower extremity muscle activity during the performance of squats and kneeling squats in persons with stroke: a preliminary study
}

\author{
Suyoung Shim ${ }^{\mathrm{a}}$, Yijung Chung ${ }^{\mathrm{b}}$ \\ ${ }^{a}$ Department of Physical Therapy, IM Convalescent Hospital, Uijeongbu, Republic of Korea \\ ${ }^{b}$ Department of Physical Therapy, College of Health Science and Social Welfare, Sahmyook University, Seoul, Republic of Korea
}

Objective: The purpose of this study was to compare the effects of performing squats and kneeling squats on trunk and lower extremity muscle activity in persons with stroke.

Design: Cross-sectional study.

Methods: Ten persons with stroke ( 3 male and 7 female) were recruited. The subjects were instructed to randomly perform the 4 different squat conditions: squat with 30 degrees of knee flexion, squat with 60 degrees of knee flexion, squat with 90 degree of knee flexion, and the kneeling squat. During the squat performance, surface electromyograms (sEMG) was used to assess muscle activity of the erector spinae (ES), gluteus maximus (Gmax), gluteus medius (Gmed), and biceps femoris (BF) muscles.

Results: Muscle activation of the ES and BF were significantly increased with the kneeling squats compared to the general squats with 30 degrees and 60 degrees of knee flexion $(p<0.05)$, and muscle activation of the Gmax and Gmed were significantly increased with the kneeling squats compared to all other squat conditions $(p<0.05)$.

Conclusions: The results suggest that the kneeling squat is an effective exercise to strengthen the proximal muscles of the lower extremities. Rather than applying a difficult general squat to the stroke population, the kneeling squat may be applied as a safer method for training the proximal muscles.

Key Words: Electromyography, Lower extremity, Stroke

\section{Introduction}

The majority of persons with stroke experience muscle weakness and movement disorders due to lesions in the descending motor pathway [1] and decreases in skeletal muscle activation [2]. As a result, due to asymmetric posture, body imbalances, and reduced weight shifting abilities, it is difficult for stroke survivors to perform functional activities [3].

In general, many stroke survivors tend to shift their center of gravity towards the paralysis side in order to compensate for limited movement and muscle weakness.
Many stroke survivors tend to have a lack of ability to produce adequate voluntary muscle contractions and are unable to perform exercises normally or maintain balance due to the inability to coordinate the timing and the intensity of muscle activity during contractions [4]. Ineffective weight shifting onto the affected side leads to sustained weakness [5]. Thus, strength training performed on the affected side can improve the functional movement of persons affected with stroke [6]. Hwang and Kim [7] lower limb muscle strength training on the paralyzed side and non-paralyzed side increases weight support rate in stroke patients. Park and Chung [8] lower limb muscle Strength training improves balance in stroke

Received: 31 May, 2019 Revised: 25 June, 2019 Accepted: 25 June, 2019

Corresponding author: Yijung Chung (ORCID https://orcid.org/0000-0002-2431-8895)

Department of Physical Therapy, College of Health Science and Social Welfare, Sahmyook University, 815 Hwarang-ro, Nowon-gu, Seoul 01795 , Republic of Korea

Tel: 82-2-3399-1637 Fax: 82-2-3399-1639 E-mail: yijung36@syu.ac.kr

(c) This is an Open-Access article distributed under the terms of the Creative Commons Attribution Non-Commercial License (http://creativecommons.org/licenses/ by-nc/4.0) which permits unrestricted non-commercial use, distribution, and reproduction in any medium, provided the original work is properly cited.

Copyright (๑) 2019 Korean Academy of Physical Therapy Rehabilitation Science 
patients. Jung et al. [9] the weight-bearing training on the paralyzed side improved gait and balance. Clinically applied squat exercises are often used for lower limb muscle training for stroke survivors. They are also used for motor control learning in nervous system rehabilitation through movement of the affected and unaffected [10]. Since squat exercise produces upper and lower body movements as well as flexion and extension of the hip, knee, and ankle joints, they are considered to be effective in improving muscle function [11] according to study Cho et al. [12]. The squat movement is a complex exercise in which not only the femoral muscle, but also the whole muscles of the trunk and lower limbs.

There have been recent studies involving squat movements and the stroke population.

A study by Gray et al. [10] included squatting at a high speed and it was suggested that the squat movement should be accompanied by the kinetic energy and speed, which are components of the force. Choi et al. [13] compared the muscle activity between the affected and unaffected sides of stroke survivors and found that squats performed at higher speeds exhibited increased muscle activity of the rectus femoris. Ki et al. [14] did a study on stroke survivors and found that modifying the squat by changing the inclination of the ankle joint of one foot to $15^{\circ}$ showed greater muscle activity of the vastus lateralis and vastus medialis when compared to performing the squat movement in the neutral state of the ankle joint and when the foot was flexed.

As shown in previous studies, research based on squat movements for the stroke population are actively being implemented, however, research on modified squat movements are insufficient. In particular, there are no studies performed that includes the squats performed while standing on the knees.

Therefore, this study was designed to investigate the effect of performing squats while standing on the knees and squats according to various knee joint angles, as well as to determine whether the modified squat movement is effective on the body and leg muscle activity. In such cases where squatting is difficult for the stroke survivor, the existing squat exercise can be modified so that it can be more easily applied within the clinical setting. This study is also an attempt to provide basic data in introducing an effective exercise method.
Table 1. General characteristics of subjects $(\mathrm{N}=10)$

\begin{tabular}{lc}
\hline Characteristic & Subjects \\
\hline Sex & $3(30.0)$ \\
Male & $7(70.0)$ \\
Female & $41.60(11.53)$ \\
Age $(\mathrm{y})$ & $166.60(7.56)$ \\
Height $(\mathrm{cm})$ & $67.00(11.46)$ \\
Weight $(\mathrm{kg})$ & $14.80(6.77)$ \\
Onset $(\mathrm{mo})$ & \\
Affected side & $5(50.0)$ \\
Right & $5(50.0)$ \\
Left & $26.20(1.95)$ \\
MMSE-K &
\end{tabular}

Values are presented as $\mathrm{n}(\%)$ or mean (SD).

MMSE-K: Korean version of the Mini-Mental State Examination.

\section{Methods}

\section{Subject}

This study is a cross-sectional study design. A total of 10 persons admitted at the IM Convalescent Hospital in Gyeonggido who were diagnosed with stroke and hemiplegia, were receiving physical therapy treatment, had met the study conditions and had agreed to participate in the study were included. The inclusion criteria for the participants were presence of hemiplegia due to stroke, the ability to walk more than $10 \mathrm{~m}$ independently without assistance, a score of 24 points or more on the Korean version of the Mini-Mental State Examination, and no visual defects or abnormalities in the vestibular system. Those who did not have orthopedic disease in the trunk and bilateral legs were selected, and those who experienced falls or trauma or pain during the past 6 months were excluded. The characteristics of the participants are as follows (Table 1).

The experiment was implemented after the experimental conditions and procedures of the study were fully explained and after the agreement to participate was obtained by the subjects. This study was approved by the research Sahmyook University Life Science ethics committee (2-7001793AB-N-012019020HS) and informed consent was obtained.

\section{Procedures}

With electrodes applied onto the erector spinae, gluteus maximus, gluteus medius, and biceps femoris muscles, the peak muscle activation values were assessed while the subjects performed squats with three different knee flexion angles as well as while standing on the knees. Prior to the experiment, the researchers explained about the squat move- 


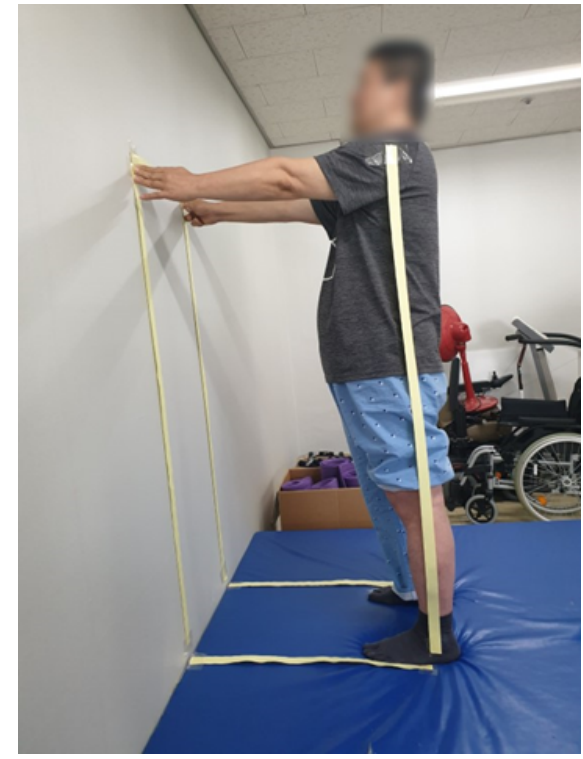

Figure 1. Guide line.

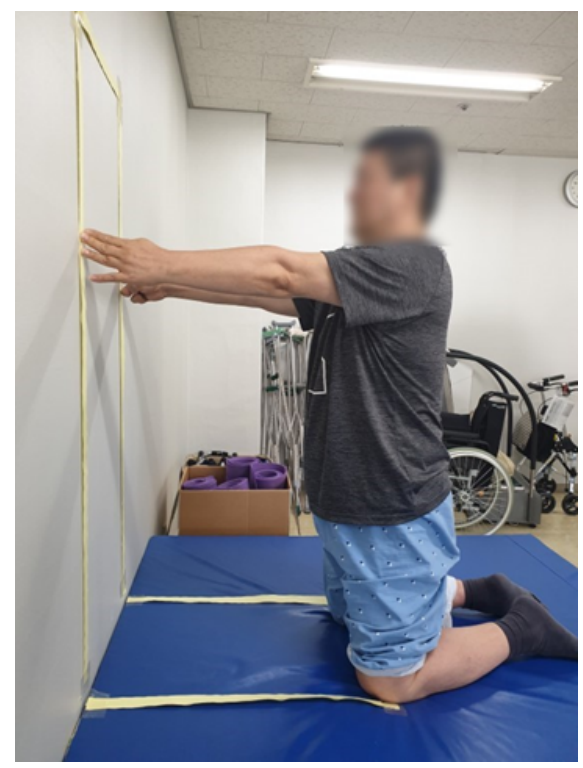

Figure 2. Kneeling position.

ment using three different knee flexion angles as well as in the standing posture of the knee, and the subjects were allowed to practice the squats more than 3 times in order to enable them to fully understand the experimental method. The squat movement was performed in four ways as stated above. In order to prevent the influence of order that the squats were performed, each type of squat was performed three times each, in random order.

The descriptions of peforming the squat movement while standing on the knees and with various knee flexion angles

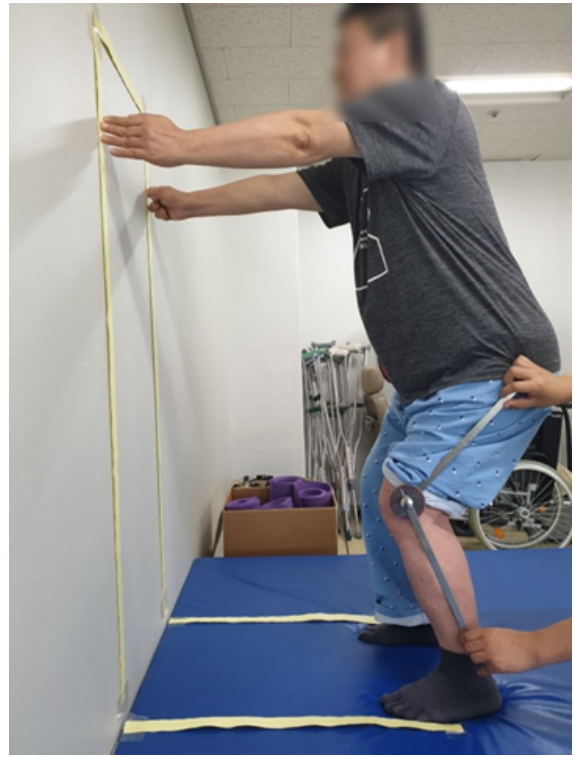

Figure 3. Squat posture.

are as follows.

The squats with use of various knee flexion angles and while standing on the knees were performed while following the guidelines that have been attached vertically onto the wall to enable the upper body to move down along with the knee flexion angles and in order to control the movement of the torso (Figure 1).

When performing the squats with use of various knee angles and while standing on the knees, the distance of the squat postures from the wall was defined as the distance from the palm and extended fingers of the unaffected side running parallel along the acromion process. During the squat movement, the feet were placed shoulder-width apart and were positioned so that the acromion process was placed vertically along the lateral malleolus.

During the performance of the squats while standing on the knees, the knees were positioned so that the acromion process and the femur were placed along a vertical line, and the legs were placed in parallel position with the knee and ankle joints aligned (Figure 2).

The knee flexion angle of the squat was set at $30^{\circ}, 60^{\circ}$, and $90^{\circ}$ using a goniometer (Baseline FEI Baseline Ss 360 Degree Goniometer; Fabrication Enterprises, Inc., White Plains, NY, USA).

The stationary arm of the goniometer was positioned parallel along the longitudinal axis of the femur while the moving arm was placed parallel along the long axis of the fibula (Figure 3). 
A 1-cm thick yoga mat was used to prevent knee pain during the performance of the squats while standing on the knees. In addition, a certain amount of knee flexion was established in order to prevent the buttocks from touching the heels (Figure 4).

While the subjects performed the squats according to various bending knee flexion angles and while standing on the knees, their muscle activation was assessed for 10 seconds total, and was repeated 3 times. Out of the 10 seconds, only data from the middle 8 seconds was used for analysis. To prevent muscle fatigue, a one minute rest period was provided between measurements.

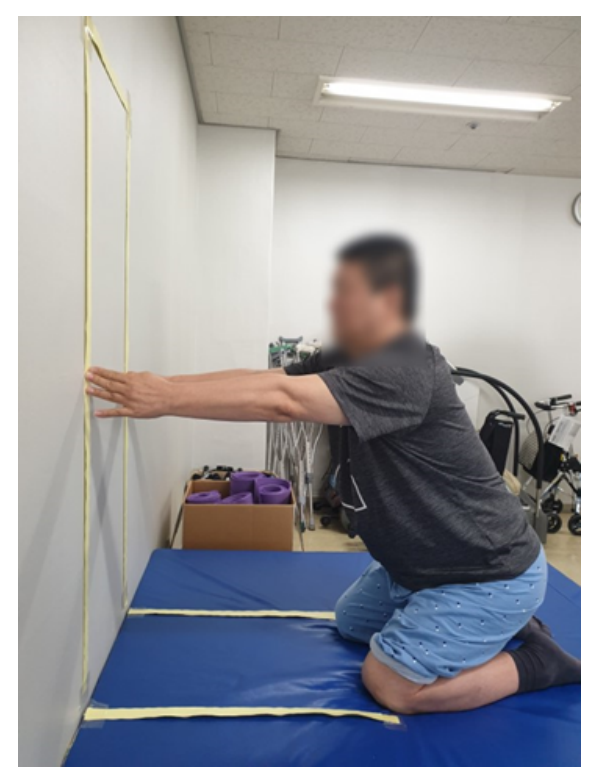

Figure 4. Kneeling squat posture.

\section{Measurement}

\section{Collection of EMG materials}

The Telemyo 2400 G2 Telemetry electromyograms (EMG) system (2011; NORAXON USA Inc., Scottsdale, AZ, USA) was used to asses the muscle activation of the erector spinae, gluteus maximus, gluteus medius, and biceps femoris muscles.

The surface EMG signal used in this study was set to a sampling rate of $1,000 \mathrm{~Hz}$ and a bandpass filter of 10 to 450 Hz. After bandpass filtering, rectification was performed and the root mean square was applied [15].

For the surface electrode $(\mathrm{Ag} / \mathrm{AgCl}$, Single Electrode $\mathrm{T} 246 \mathrm{H}$; Bioprotech, Wonju, Korea), asingle-use adhesive electrodes were used. The electrode site was removed of hair, the stratum corneum of the skin using masking sandpaper, and disinfected with alcohol swabs prior to the application of the electrodes. For the erector spinae, the surface electrode was attached along the horizontal line between the iliac crest and the third lumbar vertebrae and $2 \mathrm{~cm}$ laterally from the abdomen, for the gluteus maximus, the electrodes were attached in the center between the area inferior to the posterior superior iliac spine and the greater trochanter, for the gluteus medius, the electrodes were attached in the center of the area between the greater trochanter and the anterior superior iliac spine, and for the biceps femoris, the electrodes were attached on the area $15 \mathrm{~cm}$ inferior to the ischial tuberosities [16].

\section{Data and statistical analysis}

Nonparametric tests were used for the collected data. The Friedman test was used to compare the muscle activity of the squat movement according to various knee flexion angles $\left(30^{\circ}, 60^{\circ}\right.$, and $\left.90^{\circ}\right)$ and standing on the knee.

To determine the differences between the groups, the

Table 2. Comparison of muscle activity during squat knee angles and kneeling squat

$(\mathrm{N}=10)$

\begin{tabular}{lccccc}
\hline Condition & \multicolumn{1}{c}{$30 \mathrm{~S}$} & $60 \mathrm{~S}$ & $90 \mathrm{~S}$ & KS & $\chi^{2}(p)$ \\
\hline ES $(\mu \mathrm{V})$ & $13.50(7.03)$ & $15.21(9.54)$ & $19.15(9.28)^{\mathrm{a}, \mathrm{b}}$ & $20.06(8.33)^{\mathrm{a}, \mathrm{b}}$ & $22.280(<0.001)$ \\
GMx $(\mu \mathrm{V})$ & $3.77(2.62)$ & $3.41(1.80)$ & $4.27(2.01)$ & $6.93(2.21)^{\mathrm{a}, \mathrm{b} . \mathrm{c}}$ & $21.240(<0.001)$ \\
Gme $(\mu \mathrm{V})$ & $2.48(2.51)$ & $2.23(1.62)$ & $2.42(1.10)$ & $4.31(1.39)^{\mathrm{a}, \mathrm{b} . \mathrm{c}}$ & $15.960(0.001)$ \\
$\mathrm{BF}(\mu \mathrm{V})$ & $3.86(1.41)$ & $4.58(1.49)^{\mathrm{a}}$ & $6.25(1.60)^{\mathrm{a}, \mathrm{b}}$ & $6.47(2.78)^{\mathrm{a}, \mathrm{b}}$ & $20.520(<0.001)$ \\
\hline
\end{tabular}

Values are presented as peak $(\mu \mathrm{V})$ or mean $(\mathrm{SD})$.

30S: knee flexion $30^{\circ}$ squat, $60 \mathrm{~S}$ : knee flexion $60^{\circ}$ squat, $90 \mathrm{~S}$ : knee flexion $90^{\circ}$ squat, KS: kneeling squat, ES: elector spinae, GMx: gluteus maximus, GMe: gluteus medius, BF: bicep femoris.

${ }^{\mathrm{a}}$ Statistically significant difference with $30 \mathrm{~S}(p<0.05)$. ${ }^{\mathrm{b}}$ Statistically significant difference with $60 \mathrm{~S}(p<0.05)$. ${ }^{\mathrm{c}}$ Statistically significant difference with $90 \mathrm{~S}(p<0.05)$. 


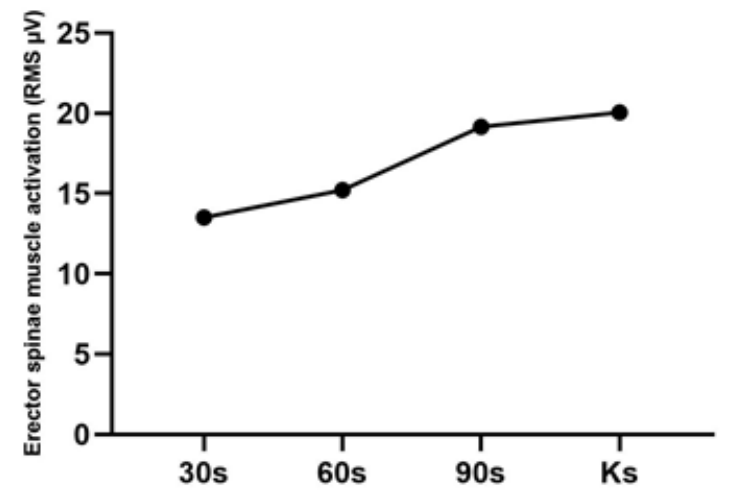

Figure 5. Erector spinae muscle activities. 30s: knee flexion 30 degree squat, 60s: knee flexion 60 degree squat, 90s: knee flexion 90 degree squat, Ks: kneeling squat.

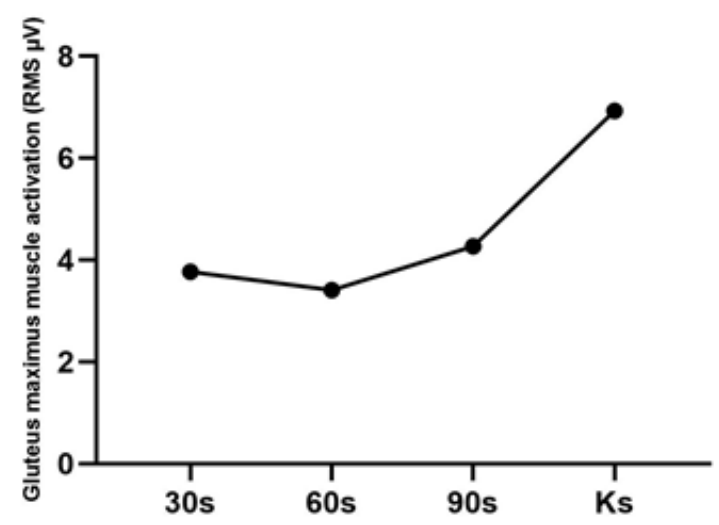

Figure 6. Gluteus maximus muscle activities. 30s: knee flexion 30 degree squat, $60 \mathrm{~s}$ : knee flexion 60 degree squat, $90 \mathrm{~s}$ : knee flexion 90 degree squat, Ks: kneeling squat.

Wilcoxon Signed-Rank test and the Bonferroni correction was used for post-hoc analysis. The SPSS Statistics for Windows, Version 20.0 (IBM Co., Armonk, NY, USA) was used for statistical processing and the significance level was set at $p<0.05$.

\section{Results}

The differences in muscle activity on the affected side while performing the squat movement according to various knee angles and while standing on the knees is as follows (Table 2).

There was a significant difference in the erector spinae, glulteus maximus, gluteus medius, and the biceps femoris muscle activity depending on the method of the squat movement according to the knee joint angle and the knee standing

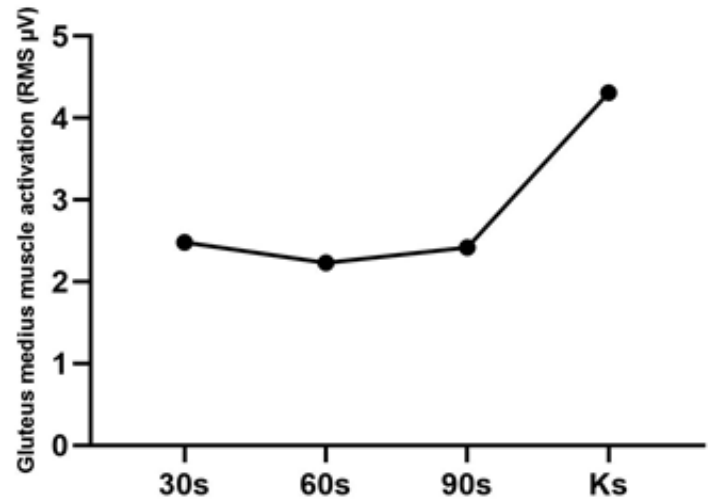

Figure 7. Gluteus medius muscle activities. 30s: knee flexion 30 degree squat, 60s: knee flexion 60 degree squat, 90s: knee flexion 90 degree squat, Ks: kneeling squat.

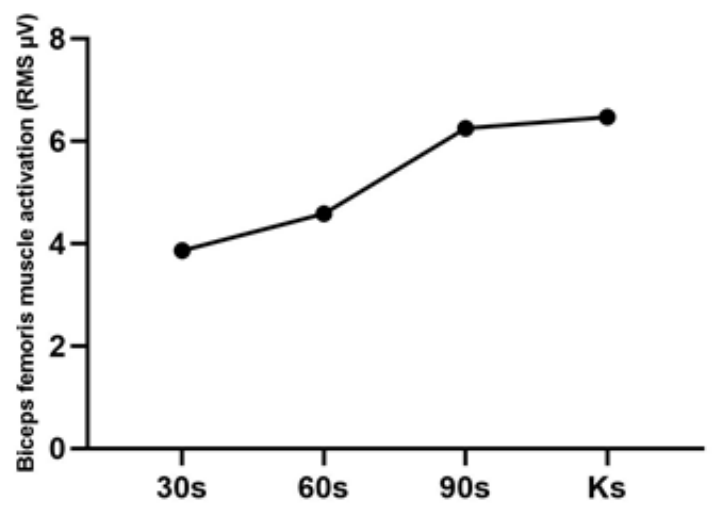

Figure 8. Biceps femoris muscle activities. 30s: knee flexion 30 degree squat, 60s: knee flexion 60 degree squat, 90s: knee flexion 90 degree squat, Ks: kneeling squat.

posture squat movement $(p<0.05)$.

\section{Post hoc Analysis Results}

Performing squats while standing on the knees and with $90^{\circ}$ of knee flexion were significantly different in the erector spinae muscle activity from that performing squats with $30^{\circ}$ and $60^{\circ}$ of knee flexion $(p<0.05)$ (Figure 5).

There was a significant difference in the gluteus maximus, gluteus medius muscle activation between the standing on the knee squats and squats performed with $30^{\circ}, 60^{\circ}$, and $90^{\circ}$ of knee flexion $(p<0.05)$ (Figures 6,7$)$.

Biceps femoris muscle activity was significantly different when performing squats with $60^{\circ}$ of knee flexion compared to $30^{\circ}$ of knee flexion $(p<0.05)$. Squats performed at $90^{\circ}$ of knee flexion was significantly different from $30^{\circ}$ and $60^{\circ}$ $(p<0.05)$ (Figure 8). 


\section{Discussion}

The purpose of this study was to investigate the effect of squat movement on the body and leg muscles when comparing the squat movement with the three knee joint flexion angles.

The results of this study showed that muscle activation while performing squats while standing on the knees and squats with $90^{\circ}$ of knee flexion were higher than when performing squats with $30^{\circ}$ and $60^{\circ}$. The squat movement performed while standing on the knees produced significantly greater muscle activation than the squat movement performed using three various knee joint flexion angles.

In other words, peak value of the proximal muscle activity involved in the squat movement while standing on the knees was similar or higher than when performing a normal squat movement.

Balance strategies include the ankle strategy, which moves the center of gravity of the body by ankle joint movements, and the hip strategy, which relocates the center of gravity by movement of the hip joints [17].

The squat movement simultaneously uses the joints of the ankle, knee, and buttock, and uses the ankle joint strategy [18]. The standing on the knee task is considered to be a developmental process and is the posture that prepares for movement and walking. The characteristics of the standing on the knees posture are that it is not possible to use an ankle strategy, and it is necessary to control to maintain the knee posture [19].

This can explain the clinical relevance of training while standing on the knees, which requires the trunk and hip strategies.

According to a study by Kurayama et al. [15], the muscle activity of the rectus abdominis, erector spinae, gluteus medius, hamstring, and rectus femoris muscle activity was higher when walking on the knees compared to the general gait within the general population. A study by Gallagher [20] investigated the changes in muscle activity and torque in the standing posture compared to standing on the knees and showed that the strength of the back and shoulder in standing posture was significantly lower than in the standing on the knees posture, which was not consistent with the results from previous studies.

This study showed that there was greater muscle activation during the squat movement when performed while standing on the knees. This suggests that the squat movement in the posture of the knee in the single-joint movement requires stronger muscular activation in the proximal part in order to produce an elongation torque, such as a general squat movement, and it is considered that the part of the body used to maintain the posture is different depending on which part of the body is being supported by the floor.

This study had a few limitations in that the sample size was small, the results cannot be compared with the unaffected side since the muscle activation of the affected side was investigated, the muscle activation was not normalized but rather the peak values were obtained. In addition, since the subjects performed 4 movements at one time the learning effect could not be completely prevented.

This study compared the squat movement while standing on the knees and the squat movement performed according to the various knee flexion angles in persons with stroke and showed that it is possible that there may be differences in muscle activity of the proximal part of the muscle. It is possible for stroke survivors to perform the squat movement safely by standing on the knees rather than performing the general squat movement, and it can be applied as a training method to improve the proximal muscle activity.

Further studies that will examine the muscle activity of the affected and unaffected side during the squat movement while standing on the knees in stroke survivors are warranted.

\section{Conflict of Interest}

The authors declared no potential conflicts of interest with respect to the authorship and/or publication of this article.

\section{References}

1. Patten C, Lexell J, Brown HE. Weakness and strength training in persons with poststroke hemiplegia: rationale, method, and efficacy. J Rehabil Res Dev 2004;41:293-312.

2. Garland SJ, Willems DA, Ivanova TD, Miller KJ. Recovery of standing balance and functional mobility after stroke. Arch Phys Med Rehabil 2003;84:1753-9.

3. Eng JJ, Chu KS. Reliability and comparison of weight-bearing ability during standing tasks for individuals with chronic stroke. Arch Phys Med Rehabil 2002;83:1138-44.

4. Olney SJ, Richards C. Hemiparetic gait following stroke. Part I: characteristics. Gait Posture 1996;4:136-48.

5. Thielman G, Kaminski T, Gentile AM. Rehabilitation of reaching after stroke: comparing 2 training protocols utilizing trunk restraint. Neurorehabil Neural Repair 2008;22:697-705.

6. Moreland JD, Goldsmith $\mathrm{CH}$, Huijbregts MP, Anderson RE, Prentice DM, Brunton KB, et al. Progressive resistance strength- 
ening exercises after stroke: a single-blind randomized controlled trial. Arch Phys Med Rehabil 2003;84:1433-40.

7. Hwang YI, Kim KS. Effects of foot pressure using the elastic band with rings during sit-to-stand in persons with stroke. Phys Ther Rehabil Sci 2017;6:159-63.

8. Park J, Chung Y. The effects of an additional weight aquatic exercise program on balance and lower extremity strength in persons with stroke: randomized controlled study. Phys Ther Rehabil Sci 2018;7:6-12.

9. Jung JH, Ko SE, Lee SW. Immediate effects of single-leg stance exercise on dynamic balance, weight bearing and gait cycle in stroke patients. Phys Ther Rehabil Sci 2014;3:49-54.

10. Gray VL, Ivanova TD, Garland SJ. Control of fast squatting movements after stroke. Clin Neurophysiol 2012;123:344-50.

11. Robertson DG, Wilson JM, St Pierre TA. Lower extremity muscle functions during full squats. J Appl Biomech 2008;24:333-9.

12. Cho M, Kang JY, Oh JH, Wu JG, Choi EB, Park SE, et al. The effects of performing squats on an inclined board on thigh muscle activation. Phys Ther Rehabil Sci 2017;6:39-44.

13. Choi YA, Kim JS, Lee DY. Effects of fast and slow squat exercises on the muscle activity of the paretic lower extremity in patients with chronic stroke. J Phys Ther Sci 2015;27:2597-9.

14. Ki KI, Choi JD, Cho HS. The effect of ground tilt on the lower extremity muscle activity of stroke patients performing squat exercises. J Phys Ther Sci 2014;26:965-8.

15. Kurayama T, Tadokoro Y, Fujimoto S, Komiya Z, Yoshida S, Chakraborty $\mathrm{S}$, et al. A comparison of the movement characteristics between the kneeling gait and the normal gait in healthy adults. Gait Posture 2013;37:402-7.

16. Cram JR, Kasman GS, Holtz J. Introduction to surface electromyography. Gaithersburg (MD): Aspen Publishers; 1998.

17. Horak FB, Shupert CL, Mirka A. Components of postural dyscontrol in the elderly: a review. Neurobiol Aging 1989;10:72738.

18. Palmitier RA, An KN, Scott SG, Chao EY. Kinetic chain exercise in knee rehabilitation. Sports Med 1991;11:402-13.

19. Dreeben-Irimia O. Physical therapy clinical handbook for PTAs. Burlington (MA): Jones \& Bartlett Publishers; 2012.

20. Gallagher S. Trunk extension strength and muscle activity in standing and kneeling postures. Spine (Phila Pa 1976) 1997; 22:1864-72. 